\title{
Homeostasis Model Assessment of Insulin Resistance in Obese Children as A Predictor for Metabolic Syndrome
}

Habiba Ahmed Abdelsalam ${ }^{1}$, Soheir Bahgat Fayed ${ }^{1}$, Amal Gaber Mohamed ${ }^{2}$, Gehan Foad Ahmed ${ }^{1}$

${ }^{1}$ Department of Pediatrics, Faculty of Medicine for Girls, Al-Azhar University, ${ }^{2}$ Department of Pediatric Nutrition, National Nutrition Institute, Cairo, Egypt

*Corresponding Author: Habiba Ahmed Abdelsalam, Phone Number: (+20) 1008925256,

Email: habibaahmed454@yahoo.com

\begin{abstract}
Background: Obesity has emerged as one of the most serious public health concerns in the 21st century. HOMAIR scores are useful indicators of insulin resistance for research purposes.

Objectives: This study was aimed to evaluate HOMA-IR for insulin resistance in obese children, to correlate between HOMA-IR and metabolic syndrome in obese children and also to assess relation between insulin resistance and morbidity. Subjects and Methods: This cross-sectional study included a total of ninety overweight and obese children, aged 4-8 years, attending at El-Zahraa University Hospital and National Institute of Nutrition. This study was conducted between from January 2018 to January 2019. All studied children were subjected to anthropometric measurement, blood pressure measuring, skin finding as acanthosis nigricans. Lab investigations including measurement of $\mathrm{HB}_{\mathrm{AlC}}$, fasting serum glucose, total cholesterol, HDL-C, LDL-C, triglycerides and insulin were done. Results: Mean and median values of HOMA-IR were significantly higher among patients with large birth weight than low and average birth weight and among patients with positive family history of obesity than negative ones. Participants illustrated a significant positive correlations between HOMA-IR and weight and BMI, SBP, DBP and serum cholesterol Conclusion: It could be concluded that homeostasis model assessment of insulin resistance index HOMA-IR in obese children is increased with increased body mass index, waist circumference systolic, diastolic blood pressure, serum cholesterol, LDL-C, large birth weight, patients with positive family history of obesity and among patients with acanthosis nigricans.
\end{abstract}

Keywords: HOMA -Insulin Resistance - Overweight and Obese children.

\section{INTRODUCTION}

Obesity has emerged as one of the most serious public health concerns in the 21 st century. The worldwide prevalence of childhood obesity has increased strikingly over the past 3 decades (1). Obesity is a proinflammatory state that increases the risk of several chronic diseases encompassing diabetes, hypertension, dyslipidemia, cardiovascular disease, asthma, sleep apnea, osteoarthritis and several cancers in adults ${ }^{(2)}$.

Measuring insulin resistance (IR), is therefore a useful tool to allow early intervention to prevent or delay the development of the disease. The gold standard method to measure IR is by hyperinsulinemic euglycemic clamp. However, the complexity and high cost of the test has prevented its use in daily clinical practice and in epidemiological studies ${ }^{(3)}$.

Homeostasis model assessment for insulin resistance (HOMA-IR) index is widely used as a measure of insulin resistance in adults and has also been validated in children and adolescents. However, the HOMA calculations require measurement of plasma fasting insulin and glucose. Due to the instability of insulin, blood collected for insulin measurement has to be kept cold, immediately processed and plasma frozen as soon as possible ${ }^{(3)}$.

HOMA-IR scores are useful indicators of insulin resistance for research purposes ${ }^{(4)}$.
This study was aimed to evaluate HOMA-IR for insulin resistance in obese children, to correlate between HOMA-IR and metabolic syndrome in obese children and also to assess relation between insulin resistance and morbidity.

\section{SUBJECTS AND METHODS}

This cross-sectional study included a total of ninety overweight and obese children, aged 4-8 years, attending at El-Zahraa University Hospital and National Institute of Nutrition. Written informed consent from all the parents of the subjects were obtained. This study was conducted between from January 2018 to January 2019.

\section{Ethical consideration:}

Approval of Research Ethics Committee of Pediatric Department and Al-Azhar Faculty of Medicine for girls was obtained.
Inclusion criteria
- Both sexes were included.
- Body mass index (BMI) z- score exceeded 2 or 3 standard deviation according to WHO BMI chart recruited for the study ${ }^{(5)}$.

\section{Exclusion criteria}
- Age less than 4 year and more than 8 years.
- obese children with: 
1. Medications causing weight gain as (sulfonylureas, cortisol, tricyclic antidepressant and monoamine oxidase inhibitors).

2. Hormonal disorders as (growth hormone deficiency or resistance, hypothyroidism, leptin deficiency or glucocorticoid excess).

3. Any infectious disease

4. Genetic disorders as (prader willi syndrome, down syndrome, turner

5. syndrome, laurance Moon Biedel syndrome)

6. Children with skeletal abnormalities and congenital malformation

7. Children with chronic disease.

8. Children with known co-morbidities such as Type 2 Diabetes mellitus, hypertension and cardiovascular disease.

Standardized data sheet appendices were utilized to record patient's history, clinical examination and investigations that were performed as follows:

1-Full history taking: age and sex, past medical history, family history, drug history and early onset of obesity.

2-Full clinical examination: Anthropometric measurement (weight, height, waist circumference)

Weight, was measured in light clothing without shoes and socks to the nearest $0.1 \mathrm{~kg}$ using a pre-calibrated body impedance analyzer (BODY COMPOSITION ANALYZER, model TBF-300).

Height: was measured without shoes to the nearest $0.1 \mathrm{~cm}$ using calibrated stadiometer (RAVEN EQUIPMENT LIMITED , ENGLAND).

Body Mass Index (BMI): $\quad$ BMI $\quad$ - score According to WHO BMI chart ${ }^{(5)}$.

Overweight: Defined as BMI z-score $\geq 2$ SD.

Obesity: Defined as BMI z-score $\geq 3$ SD.

Waist Circumference: was measured at the minimum circumference between the iliac crest and the rib cage using a tape measure ${ }^{(\boldsymbol{6})}$.

Blood pressure (systolic and diastolic blood pressure) measurement. Two readings of blood pressure was measured after 5 minutes of resting in sitting position using a mercury sphygmomanometer (ALPK2 , JAPAN) ${ }^{(7)}$.

Examination of Acanthosis Nigricans (AN) (Acanthosis nigricans was a brown to black, poorly, velvety hyperpigmentation of the skin. It is usually found in body folds such as the posterior and lateral folds of the neck, the armpits, groin, navel, forehead, and other areas. It's most common in those who are overweight, have darker skin, and have diabetes or prediabetic conditions) ${ }^{(\mathbf{8})}$.
3-Laboratory investigations: Fasting serum glucose, insulin, total cholesterol, HDL-C, LDL-C and triglycerides were measured.

Sampling: Fasting venous blood samples were obtained and distributed between EDTA containing tube for measuring $\mathrm{HB}_{\mathrm{AlC}}$ and plain tubes for separation of serum to measure glucose, total cholesterol, HDL-C, LDL-C, triglycerides and insulin.

- HOMA will be calculated by multiplying the value of fasting insulin and fasting glucose and divided by 22.5. The score of $\geq 3.5$ was classified as insulin resistance, while a score of less than 3.5 was considered as insulin sensitive ${ }^{(7)}$.

HOMA-IR= Glucose $\mathrm{x}$ Insulin / (Glucose in molar unit $\mathrm{mmol} / \mathrm{l}$ )

\section{5}

Metabolic syndrome will be considered present if the waist circumference measurement was $\geq 90$ thcentile of the waist circumference chart .with the presence of at least two of the following criteria; triglycerides $\geq 1.7 \mathrm{mmol} / \mathrm{L}, \quad \mathrm{HDL}$ cholesterol $<1.03 \mathrm{mmol} / \mathrm{L}, \quad$ systolic blood pressure $\geq 130 \mathrm{mmHg}$ and/or diastolic blood pressure $\geq 85 \mathrm{mmHg}$, or fasting plasma glucose $\geq 5.6 \mathrm{mmol} / \mathrm{L}^{(9)}$.

\section{Statistical analysis}

The collected data were tabulated and analyzed using SPSS version 18 soft ware (Spss Inc, Chicago, ILL Company). Categorical data were presented as number and percentages. Chi square test $\left(\mathrm{X}^{2}\right)$, or Fisher's exact test (FET) were used to analyze categorical variables. Quantitative data were tested for normality using Shapiro-Wilks test, assuming normality at $\mathrm{P}>0.05$, they were expressed as mean \pm standard deviation if normally distributed or median and IQR if not. Student "t" test was used to analyze normally distributed variables among 2 independent groups. While non parametric variables were analyzed using Man Whitney U test. Spearman's correlation coefficient (rho) was used to assess liner associations between variables. The accepted level of significance in this work was stated at $0.05(\mathrm{P}<0.05$ was considered significant). $P$ value $>0.05$ is non significant (NS) $P<0.05$ is significant $(S) . P \leq 0.001$ is highly significant $(H S)$.

\section{RESULTS}

Table (1) shows that there was no statistically significant difference regarding sex or educational level ( $\mathrm{P}>0.05$ for both), while it was significant to birth weight $(\mathrm{P}<0.05)$.

Table (2) shows that there was no statistically significant difference regarding history of asthma, physical activity or easily fatigability ( $\mathrm{P}>0.05$ for all). 
while it was significant among patients with positive family history of obesity $(\mathrm{P}<0.05)$.

Table (3) shows that there was no statistically significant difference in the level of HOMA-IR regarding sleep disorders $(\mathrm{P}>0.05)$ while it was significant among patients with acanthosis nigricans $(\mathrm{P}<0.05)$.

Table (4) shows that there were significant positive correlations between HOMA-IR and weight and BMI $(p=0.002$ and $<0.001$ respectively). On the other hand, its correlations with age and length were non significant ( $\mathrm{P}>0.05$ for both).

Table (5) illustrates that there was significant positive correlations between HOMA-IR and waist circumference and $\mathrm{W} / \mathrm{H}$ ratio $(\mathrm{P}<0.05$ for both). On the other hand, its correlations with hip circumference and triceps skin fold thickness were non significant ( $\mathrm{P}>0.05$ for both).
Table (6) illustrates that there were significant positive correlations between HOMA-IR and $\mathrm{S}$ cholesterol and LDL $(\mathrm{P}<0.05$ for both).While, its correlations with $\mathrm{S}$ triglycerides and HDL were nonsignificant ( $\mathrm{P}>0.05$ for both).

Table (7) shows Prevalence of HOMA IR among the studied sample was $(14.4 \%)$, prevalence of WC centiles $>90$ centiles was $(71.1 \%)$, prevalence of BP centile $>90$ centiles was $(68.9 \%)$, prevalence of dyslipidemia was (53.3\%).

Regarding WC, it was found that they were statistically significantly higher among insulin resistance group than non-insulin resistance group. BP centile was significantly higher among insulin resistance group than non insulin resistance group.Dyslipidemia was significantly higher among insulin resistance group than non insulin resistance group Table (8).

Table (1): Relation between socio demographic characters and HOMA IR level

\begin{tabular}{|c|c|c|c|c|c|c|c|c|}
\hline & & \multicolumn{5}{|c|}{ HOMA IR } & \multirow[b]{2}{*}{$\mathbf{Z}_{\mathbf{M W U}}$} & \multirow[b]{2}{*}{ P value } \\
\hline & & Mean & $\begin{array}{l}\text { Standard } \\
\text { Deviation }\end{array}$ & Median & Minimum & Maximum & & \\
\hline \multirow{2}{*}{ Sex } & $\mathbf{M}$ & 2.02 & 1.52 & 1.58 & .70 & 9.76 & \multirow{2}{*}{-0.569} & \multirow{2}{*}{0.569} \\
\hline & $\mathbf{F}$ & 2.12 & 1.38 & 1.55 & .70 & 7.34 & & \\
\hline \multirow{2}{*}{ education of mother } & low & 2.05 & 1.41 & 1.58 & .70 & 9.76 & \multirow{2}{*}{-0.044} & \multirow{2}{*}{0.965} \\
\hline & high & 2.12 & 1.57 & 1.56 & .79 & 7.34 & & \\
\hline \multirow{3}{*}{ birth weight } & low & 1.37 & .57 & 1.08 & .76 & 2.33 & \multirow{3}{*}{$\begin{array}{l}\mathrm{KW} \\
=8.31\end{array}$} & \multirow{3}{*}{0.016} \\
\hline & average & 1.94 & 1.36 & 1.50 & .70 & 9.76 & & \\
\hline & large & 2.78 & 1.74 & 2.28 & .70 & 7.34 & & \\
\hline
\end{tabular}

$Z_{\mathrm{MWU}} \rightarrow \mathrm{Z}$ value of Mann Whitney $\mathrm{U}$ test $\mathrm{KW}=$ Kruskal Wallis test

Table (2): Relation between history, lifestyle and HOMA IR level

\begin{tabular}{|c|c|c|c|c|c|c|c|c|}
\hline & & \multicolumn{5}{|c|}{ HOMA IR } & \multirow[b]{2}{*}{$\mathbf{Z}_{\mathbf{M W U}}$} & \multirow[b]{2}{*}{ P value } \\
\hline & & Mean & $\begin{array}{l}\text { Standard } \\
\text { Deviation }\end{array}$ & Median & Minimum & Maximum & & \\
\hline \multirow{2}{*}{$\begin{array}{l}\text { family history of } \\
\text { obesity }\end{array}$} & yes & 2.59 & 1.80 & 2.27 & 0.70 & 9.76 & \multirow{2}{*}{3.162} & \multirow{2}{*}{0.002} \\
\hline & no & 1.60 & .78 & 1.39 & 0.76 & 4.53 & & \\
\hline \multirow{2}{*}{ history of asthma } & yes & 2.35 & 1.66 & 1.90 & 0.76 & 7.34 & \multirow{2}{*}{-0.748} & \multirow{2}{*}{0.455} \\
\hline & no & 1.96 & 1.35 & 1.56 & 0.70 & 9.76 & & \\
\hline \multirow{2}{*}{ physical activity } & yes & 2.01 & 1.16 & 1.64 & 0.79 & 5.56 & \multirow{2}{*}{0.246} & \multirow{2}{*}{0.806} \\
\hline & no & 2.10 & 1.58 & 1.52 & 0.70 & 9.76 & & \\
\hline \multirow{2}{*}{ easily fatigability } & yes & 2.14 & 1.75 & 1.51 & .70 & 9.76 & \multirow{2}{*}{0.585} & \multirow{2}{*}{0.558} \\
\hline & no & 2.00 & 1.06 & 1.74 & .70 & 5.56 & & \\
\hline
\end{tabular}

Table (3): Relation between sleeping disorder, acanthosis nigra and HOMA IR level 


\begin{tabular}{|c|c|c|c|c|c|c|c|c|}
\hline & & \multicolumn{5}{|c|}{ HOMA IR } & \multirow[b]{2}{*}{$\mathbf{Z}_{\mathbf{M W U}}$} & \multirow[b]{2}{*}{ P value } \\
\hline & & Mean & \begin{tabular}{|l|} 
Standard \\
Deviation
\end{tabular} & Median & Minimum & Maximum & & \\
\hline \multirow{2}{*}{ sleep disorder } & yes & 2.30 & 1.85 & 1.57 & .76 & 9.76 & \multirow{2}{*}{-0.506} & \multirow{2}{*}{0.613} \\
\hline & no & 1.90 & 1.06 & 1.57 & .70 & 5.56 & & \\
\hline \multirow{2}{*}{ acanthosis nigercans } & yes & 2.45 & 1.70 & 1.76 & 0.98 & 9.76 & \multirow{2}{*}{-3.128} & \multirow{2}{*}{$0.02(\mathrm{~S})$} \\
\hline & no & 1.53 & 0.71 & 1.44 & 0.70 & 4.01 & & \\
\hline
\end{tabular}

Table (4): Correlation between HOMA IR and anthropometric measures

\begin{tabular}{|l|l|l|}
\hline \multirow{4}{*}{ Age (years) } & & HOMA IR \\
\hline \multirow{5}{*}{ Weight $(\mathbf{k g})$} & Correlation Coefficient & $-.006-$ \\
\cline { 2 - 3 } & P value & .959 \\
\cline { 2 - 3 } & $\mathbf{N}$ & 90 \\
\hline \multirow{3}{*}{ Hight $(\mathbf{c m})$} & Correlation Coefficient & 0.323 \\
\cline { 2 - 3 } & P value & 0.002 \\
\hline & $\mathbf{N}$ & 90 \\
\hline \multirow{3}{*}{ BMI $\left(\mathbf{k g} / \mathbf{m}^{\mathbf{2}}\right)$} & Correlation Coefficient & $-.006-$ \\
\cline { 2 - 3 } & P value & .954 \\
\hline & $\mathbf{N}$ & 90 \\
\hline & Correlation Coefficient & 0.440 \\
\hline & P value & $<0.001$ \\
\hline & $\mathbf{N}$ & 90 \\
\hline
\end{tabular}

Table (5): Correlation between HOMA IR and anthropometric measurement

\begin{tabular}{|l|l|l|}
\hline & & HOMA IR \\
\hline \multirow{4}{*}{ waist circumference } & Correlation Coefficient & 0.371 \\
\cline { 2 - 3 } & P value & $<0.001(\mathrm{HS})$ \\
\cline { 2 - 3 } & $\mathbf{N}$ & 90 \\
\hline \multirow{5}{*}{ hip circumference } & Correlation Coefficient & .072 \\
\cline { 2 - 3 } & $\mathbf{P}$ value & .498 \\
\cline { 2 - 3 } & $\mathbf{N}$ & 90 \\
\hline \multirow{4}{*}{ W/H ratio } & Correlation Coefficient & 0.279 \\
\cline { 2 - 3 } & $\mathbf{P}$ value & 0.009 \\
\cline { 2 - 3 } & $\mathbf{N}$ & 76 \\
\hline \multirow{4}{*}{ triceps skin fold } & Correlation Coefficient & .091 \\
\cline { 2 - 3 } & P value & .392 \\
\cline { 2 - 3 } & $\mathbf{N}$ & 90 \\
\hline
\end{tabular}


Table (6): Correlation between HOMA IR and lipid profile among cases

\begin{tabular}{|l|l|l|}
\hline \multirow{4}{*}{ Cholesterol } & & HOMA IR \\
\hline \multirow{4}{*}{ Triglycerides } & Correlation Coefficient & .256 \\
\cline { 2 - 3 } & P value & 0.015 \\
\cline { 2 - 3 } & $\mathbf{N}$ & 90 \\
\hline \multirow{3}{*}{ HDL-C } & Correlation Coefficient & .053 \\
\cline { 2 - 3 } & P value & .622 \\
\cline { 2 - 3 } & $\mathbf{N}$ & 90 \\
\hline \multirow{3}{*}{ LDL-C } & Correlation Coefficient & $-.132-$ \\
\cline { 2 - 3 } & P value & .215 \\
\hline & $\mathbf{N}$ & 90 \\
\hline & Correlation Coefficient & .314 \\
\cline { 2 - 3 } & P value & .003 \\
\cline { 2 - 3 } & $\mathbf{N}$ & 90 \\
\hline
\end{tabular}

Table (7): Description (count and \% in the study group)

\begin{tabular}{|c|c|c|c|}
\hline & & Count & $\%$ \\
\hline \multirow{2}{*}{ HOMA IR } & $>3.5$ & 13 & $14.4 \%$ \\
\hline & $<3.5$ & 77 & $85.6 \%$ \\
\hline \multirow{2}{*}{ F sugar $>100$} & Yes & 18 & $20.0 \%$ \\
\hline & No & 72 & $80.0 \%$ \\
\hline \multirow{2}{*}{ waist circumference centile } & $>90$ percentile & 64 & $71.1 \%$ \\
\hline & $<90$ percentile & 26 & $28.9 \%$ \\
\hline \multirow{2}{*}{ BP centile } & $>90$ percentile & 62 & $68.9 \%$ \\
\hline & $<90$ percentile & 28 & $31.1 \%$ \\
\hline \multirow{2}{*}{ dyslipidemia } & Yes & 48 & $53.3 \%$ \\
\hline & No & 42 & $46.7 \%$ \\
\hline
\end{tabular}

Table (8): Relation between HOMA IR and other components of metabolic syndrome

\begin{tabular}{|c|c|c|c|c|c|c|}
\hline & \multicolumn{4}{|c|}{ HOMA IR } & \multirow{3}{*}{ P value } \\
\hline & & \multicolumn{2}{|l|}{$>3.5$} & \multicolumn{2}{|l|}{$<3.5$} & \\
\hline & & Count & N\% & Count & $\%$ & \\
\hline \multirow{2}{*}{ F Sugar $>100$} & Yes & 11 & $84.6 \%$ & 7 & $9.1 \%$ & \multirow[t]{2}{*}{$<0.001$} \\
\hline & No & 2 & $15.4 \%$ & 70 & $90.9 \%$ & \\
\hline \multirow{2}{*}{$\begin{array}{l}\text { waist circumference } \\
\text { centile }\end{array}$} & $>90$ percentile & 13 & $100.0 \%$ & 51 & $66.2 \%$ & \multirow[t]{2}{*}{0.017} \\
\hline & $<90$ percentile & 0 & $0.0 \%$ & 26 & $33.8 \%$ & \\
\hline \multirow{2}{*}{ BP centile } & $>90$ percentile & 13 & $100.0 \%$ & 49 & $63.6 \%$ & \multirow[t]{2}{*}{0.008} \\
\hline & $<90$ percentile & 0 & $0.0 \%$ & 28 & $36.4 \%$ & \\
\hline \multirow{2}{*}{ dyslipidemia } & Yes & 12 & $92.3 \%$ & 36 & $46.8 \%$ & \multirow{2}{*}{0.002} \\
\hline & No & 1 & $7.7 \%$ & 41 & $53.2 \%$ & \\
\hline
\end{tabular}




\section{DISCUSSION}

This study showed that, there was no statistically significant difference in the level of HOMA-IR regarding sex $(\mathrm{P}>0.05)$. This agreed with the result of the study done by ${ }^{\left({ }^{(10)}\right.}$ who found that, there was no statistically significant difference between sex and level of HOMA-IR.

Lentferink et al. ${ }^{(11)}$ reported that in children, girls were significantly more diagnosed with IR. This is in concordance with recent literature; in addition, a higher prevalence of IR in girls without obesity is described ${ }^{(\mathbf{1 2})}$. This result can be explained by the fact that pubertal development starts earlier in girls. In adolescents, no difference in gender was observed, possibly because the degree of obesity blurred the effect of puberty as described earlier.

This study showed that, mean and median values of HOMA-IR were significantly higher among patients with large birth weight. These results was nearly agreed with Lentferink et $\boldsymbol{a l}$. $^{(11)}$. As regard family history of obesity the study showed that, mean and median values of HOMA-IR were significantly higher among patients with positive family history of obesity than negative ones This is similar to the results of the study done by Corica et al. ${ }^{(13)}$ who reported that a family history for obesity is an important risk factor for precocious obesity onset in childhood and influences the severity of obesity; and metabolic profile, especially HOMA-IR, is altered even among the youngest obese children at first evaluation.

When we studied the mean value of HOMA-IR among patients with acanthosis nigricans it was significantly higher when compared to those without than without acanthosis nigricans (2.45 and 1.53 respectively) $\mathrm{P}<0.05$. This agreed with Koh et al. ${ }^{\text {(14) }}$ who found that, the With acanthosis nigricans group had higher HOMA-IR score $(5.74 \pm 4.71$ vs. $2.14 \pm 0.86, \quad \mathrm{p}<0.001)$ than Without acanthosis nigricans group. HOMA-IR scores increased with acanthosis nigricans severity, from the without acanthosis nigricans group. Insulin resistance worsens with increasing acanthosis nigricans severity, and patients with Severe acanthosis nigricans (acanthosis nigricans score $\geq 3$ ) are at increased risk of insulin resistance.

Acanthosis nigricans is known to occur when the concentration of insulin-like growth factor receptors in the skin is too low relative to the amount of insulin present, causing accumulation of insulin in the skin, proliferation of epidermal cells, and thickening of keratocytes acanthosis nigricans is associated with obesity and endocrine diseases caused by severe insulin resistance, and may rarely be seen in patients with genetic diseases or malignant tumors ${ }^{(15)}$.

The development of insulin resistance in obese patients can cause several metabolic abnormalities to occur simultaneously. This phenomenon was first named "syndrome X" by Reaven ${ }^{(16)}$. Since then, various studies have demonstrated that insulin resistance can cause dyslipidemia, fatty liver disease, hypertension, and type 2 diabetes, and this phenomenon is now more commonly referred to as "metabolic syndrome" (17).

Kahn et al. ${ }^{(18)}$ first suggested an association between acanthosis nigricans and insulin resistance in obese children in 1976. Since then, many studies have been conducted with the aim of utilizing acanthosis nigricans as an indicator for insulin resistance $^{(\mathbf{1 9})}$.

In our studied participants illustrated that there were significant positive correlations between HOMA-IR and weight and BMI ( $\mathrm{p}=0.002$ and $<0.001$ respectively).

Vrablík et al. ${ }^{(20)}$ demonstrated the correlation of AIP with BMI and HOMA-IR in children.

This similar to the result of the study done by Iwani et al. ${ }^{(1)}$ who found that, BMI was statistically significantly higher among insulin resistance group.

This agreed also with Romualdo et al. ${ }^{(21)}$ who found, there were significant positive correlations between HOMA-IR and weight and BMI.

As regard systolic and diastolic blood pressure there were significant positive correlations between HOMA-IR and SBP and DBP ( $\mathrm{P}<0.001$ for both).

This agreed with Barseem and Helwa ${ }^{(22)}$ who found that, the correlation was strongest with body mass index, systolic and diastolic blood pressure.

Our study results revealed significant positive correlations between HOMA-IR and waist circumference and $\mathrm{W} / \mathrm{H}$ ratio $(\mathrm{P}<0.05$ for both). This agreed with Bacopoulou et al. ${ }^{(23)}$ who found that, central obesity is one of the strongest indicators of insulin resistance. He stated that WC, WHR and WHtR are the useful measurements for central obesity, and WC is the most sensitive index. This could be explained by that individuals with higher WC have more visceral fat and are more susceptible to chronic diseases caused by insulin resistance than those with lower WC and the same BMI ${ }^{(24)}$.

Korean studies Kim et al ${ }^{(25)}$ and Kang et al. ${ }^{(26)}$ adopted the stratification of WC to dispel its effect on insulin resistance as well. One also had exceptional conditions which were analogous to ours.

Insulin resistance influences the link between obesity and dyslipidemia. Vukovic et al. (27) 
demonstrated that HOMA-IR was strictly related to lipid profile in their pediatric population.

As regard lipid profile among studied group there were no significant correlations between HOMA-IR and S triglycerides and HDL.

This agreed with Barseem and Helwa ${ }^{(22)}$ who found that, there were no significant correlations between HOMA-IR and S triglycerides and HDL ( $\mathrm{P}>0.05$ for both).

In this study there were significant positive correlations between HOMA-IR and S cholesterol and LDL ( $\mathrm{P}<0.05$ for both).

This agreed with Barseem and Helwa ${ }^{(22)}$ who found that, study there were significant positive correlations between HOMA-IR and S cholesterol and LDL.

Our study result revealed that, prevalence of HOMA IR among the studied sample was (14.4\%). This is lower than study done by Iwani et al. ${ }^{(1)}$ who found a total of 271 blood samples of obese and overweight children aged 9-16 years of whom 49\% children were found to have IR.

Our study result showed that, prevalence of BP centile $>90$ centiles was $(68.9 \%)$.

Obesity influences BP in children. In a large cohort study of more than 100,000 children and adolescents followed for several years, those with obesity and severe obesity had higher BP at baseline and a greater odd of developing hypertension years later than those of lower BMI categories ${ }^{(28)}$.

Our study result showed that, prevalence of dyslipidemia was $(53.3 \%)$.This agreed with Elmaoğulları et al. ${ }^{(29)}$ who found that, among 823 obese patients, 353 (42.9\%) had dyslipidemia.

Regarding WC, it was found that they were statistically significantly higher among insulin resistance group than non-insulin resistance group.

Bacopoulou et al. ${ }^{(23)}$ found that, central obesity is one of the strongest indicators of insulin resistance. He stated that WC, WHR and WHtR are the useful measurements for central obesity, and WC is the most sensitive index.

It has been reported that individuals with higher WC have more visceral fat and are more susceptible to chronic diseases caused by insulin resistance than those with lower WC and the same BMI ${ }^{(\mathbf{1})}$.

In this study BP centile was significantly higher among insulin resistance group than non insulin resistance group.

The same observation was reported by Armato et al. ${ }^{\left({ }^{30)}\right.}$ who revealed that individuals with insulin resistance had significantly higher BP.

This study showed that, dyslipidemia was significantly higher among insulin resistance group than non-insulin resistance group.
This is similar to the results of the study done by Kim-Dorner et al. ${ }^{(3)}$ who found that, IR was associated with dyslipidemia.

As shown in many studies, hypertriglyceridemia and decreased HDL-C are the hallmarks of dyslipidemia which is a characteristic of insulin resistance and T2D. The Treatment Options for Type 2 Diabetes in Adolescents and Youth (TODAY) study showed that $79.8 \%$ of T2D youth had a low HDL-C and $10.2 \%$ had high triglycerides within a few months of diagnosis and the SEARCH for Diabetes in Youth study found that 73\% of 2096 US youth with T2D of longer duration had lower HDL and $60-65 \%$ had hypertriglyceridemia ${ }^{(31)}$.

Therefore, the clinical utility of measuring TG and HDL-C extended beyond identifying patients with IR ${ }^{(21)}$.

\section{CONCLUSION}

It could be concluded that homeostasis model assessment of insulin resistance index HOMA-IR in obese children were increased with increased body mass index, waist circumference systolic, diastolic blood pressure, serum cholesterol, LDL, large birth weight, patients with positive family history of obesity and among patients with acanthosis nigricans.

Sources of Support: No funding - No grants. Conflict of Interest: No conflict of interest.

\section{REFERENCES}

1. Iwani NAKZ, Jalaludin MY et al. (2017): Triglyceride to HDL-C Ratio is Associated with Insulin Resistance in Overweight and Obese Children. Sci Rep., 7:40055.

2. Renzaho AMN, Green, J, Smith BJ et al. (2017): Immigrant Minority Health. https://doi.org/10.1007/s10903-017-0620-6

3. Kim-Dorner SJ, Deuster PA, Zeno SA(2010): Should triglycerides and the triglycerides to high-density lipoprotein cholesterol ratio be used as surrogates for insulin resistance? Metabolism: Clinical and Experimental, 59(2):299-304.

4. Hirschler V, Maccallini G, Sanchez M et al. (2015): Association between triglyceride to HDL-C ratio and insulin resistance in indigenous Argentinean children. Pediatric Diabetes, 16: 606-612.

5. Collins K, Sharif B, Sanmartin $\mathrm{C}$ et al. (2017): Association of body mass index (BMI) and percent body fat among BMI-defined non-obese middle-aged individuals: Insights from a population-based Canadian sample. Can J Public Health.6: 520.

6. Seidell JC, Pérusse L, Després JP et al. (2001b): Waist and hip circumferences have independent and opposite effects on cardiovascular disease risk factors: the Quebec Family Study. Am J Clin Nutr., 74: 315-21.

7. Tamura T, Mizukura I, Sekine $M$ et al. (2011): Monitoring and evaluation of blood pressure changes with 
a home healthcare system. IEEE Transactions on Information Technology in Biomedicine, 15(4): 602- 607.

8. Klish WJ (2013): Clinical evaluation of the obese child and adolescent. In:Motil KJ, Geffner M (section eds) and Hoppin AG (Deputy ed): Up to date. www.uptodate.com@UpToDate.

9. Kawada T, Andou T, Fukumitsu M (2016):Waist circumference, visceral abdominal fat thickness and three components of metabolic syndrome. Diabetes \& Metabolic Syndrome: Clinical Research \& Reviews, 1(10): 4-6.

10. Matone A, Derlindati E, Marchetti L et al. (2017): Identification of an early transcriptomic signature of insulin resistance and related diseases in lymphomonocytes of healthy subjects. PLoS ONE, 12(8): e0182559.

11. Lentferink YE, Elst M, Knibbe C et al. (2017): Predictors of Insulin Resistance in Children versus Adolescents with Obesity. Journal of Obesity, 2017: 3793868 .

12. van der Aa MP, Fazeli Farsani S, Knibbe CA et al. (2015): Population-based studies on the epidemiology of insulin resistance in children. Journal of Diabetes Research, 2015:9.

13. Corica D, Aversa T, Valenzise M et al. (2018): Does Family History of Obesity, Cardiovascular, and Metabolic Diseases Influence Onset and Severity of Childhood Obesity?. Frontiers in Endocrinology, 9: 187.

14. Koh YK, Lee JH, Kim EY et al. (2016): Acanthosis Nigricans as a Clinical Predictor of Insulin Resistance in Obese Children. Pediatric Gastroenterology, Hepatology \& Nutrition, 19(4): 251-258.

15. Hermanns-Lê T, Scheen A, Piérard GE (2004): Acanthosis nigricans associated with insulin resistance: pathophysiology and management. Am J Clin Dermatol., 5:199-203.

16. Reaven GM (1993): Role of insulin resistance in human disease (syndrome X): an expanded definition. Annu Rev Med., 44:121-131.

17. Hong YM (2009): Metabolic syndrome in children and adolescents. Korean J Pediatr., 52:737-744.

18. Kahn CR, Flier JS, Bar RS et al. (1976): The syndromes of insulin resistance and acanthosis nigricans. Insulinreceptor disorders in man. N Engl J Med., 294:739-745.

19. Chueh HW, Cho GR, Yoo JH (2007): Clinical significance of acanthosis nigricans in children and adolescents with obesity induced metabolic complications. Korean J Pediatr., 50:987-994.

20. Vrablík M, Dobiášová M, Zlatohlávek L et al. (2014): Biomarkers of cardiometabolic risk in obese/overweight children: effect of lifestyle intervention. Physiol Res., 63(6):743-52.
21. Romualdo MC, de Nóbrega FJ, Escrivão MAMS et al. (2014): Insulin resistance in obese children and adolescents. Jornal de Pediatria, 90(6): 600-607.

22. Barseem NF, Helwa MA (2015): Homeostatic model assessment of insulin resistance as a predictor of metabolic syndrome: Consequences of obesity in children and adolescents. Egyptian Pediatric Association Gazette, 63(1): 19-24.

23. Bacopoulou F, Efthymiou V, Landis G et al. (2015): Waist circumference, waist-to-hip ratio and waist-toheight ratio reference percentiles for abdominal obesity among Greek adolescents. BMC Pediatrics, 15:50-5.

24. Tanaka S, Horimai C, Katsukawa F (2003): Ethnic differences in abdominal visceral fat accumulation between Japanese, African-Americans, and Caucasians: a meta-analysis. Acta diabetologica, 40(1):302-4.

25. Kim JS, Kang HT, Shim JY et al. (2012): The association between the triglyceride to high-density lipoprotein cholesterol ratio with insulin resistance (HOMA-IR) in the general Korean population: based on the National Health and Nutrition Examination Survey in 2007-2009. Diabetes Research and Clinical Practice, 97(1):132-8.

26. Kang HT, Yoon JH, Kim JY et al. (2012): The association between the ratio of triglyceride to HDL-C and insulin resistance according to waist circumference in a rural Korean population. Nutrition, metabolism, and cardiovascular diseases: NMCD., 22(12):1054-60.

27. Vukovic $\mathbf{R}$, Mitrovic $\mathrm{K}$, Milenkovic $\mathrm{T}$ et al. (2013):Insulin-sensitive obese children display a favorable metabolic profile. Eur J Pediatr., 172:201-6.

28. Brady TM (2017): Obesity-Related Hypertension in Children. Frontiers in Pediatrics, 5: 197-203.

29. Elmaoğulları S, Tepe D, Uçaktürk SA et al. (2015): Prevalence of Dyslipidemia and Associated Factors in Obese Children and Adolescents. Journal of Clinical Research in Pediatric Endocrinology, 7(3):228-234.

30. Armato J, Reaven G, Ruby R et al. (2015): Triglyceride/High-Density Lipoprotein Cholesterol Concentration Ratio Identifies Accentuated CardioMetabolic Risk. Endocrine practice: official journal of the American College of Endocrinology and the American Association of Clinical Endocrinologists, 21: 1-18.

31. Copeland, K. C, Zeitler, P, Sanmartin C et al. (2011): Characteristics of Adolescents and Youth with RecentOnset Type 2 Diabetes: The TODAY Cohort at Baseline. The Journal of Clinical Endocrinology and Metabolism, 96(1): 159-167.

32. Lee JM, Okumura MJ, Davis MM et al. (2006): Prevalence and determinants of insulin resistance among US adolescents: a population-based study. Diabetes Care, 29: 2427-2432. 